\title{
OPTIMISATION OF TIME-SCHEDULED REGIMEN FOR ANTI-CANCER DRUG INFUSION
}

\author{
Claude Basdevant $^{1}$, Jean Clairambault ${ }^{2}$ and Francis Lévi ${ }^{3}$
}

\begin{abstract}
The chronotherapy concept takes advantage of the circadian rhythm of cells physiology in maximising a treatment efficacy on its target while minimising its toxicity on healthy organs. The object of the present paper is to investigate mathematically and numerically optimal strategies in cancer chronotherapy. To this end a mathematical model describing the time evolution of efficiency and toxicity of an oxaliplatin anti-tumour treatment has been derived. We then applied an optimal control technique to search for the best drug infusion laws. The mathematical model is a set of six coupled differential equations governing the time evolution of both the tumour cell population (cells of Glasgow osteosarcoma, a mouse tumour) and the mature jejunal enterocyte population, to be shielded from unwanted side effects during a treatment by oxaliplatin. Starting from known tumour and villi populations, and a time dependent free platinum $P t$ (the active drug) infusion law being given, the mathematical model allows to compute the time evolution of both tumour and villi populations. The tumour population growth is based on Gompertz law and the Pt anti-tumour efficacy takes into account the circadian rhythm. Similarly the enterocyte population is subject to a circadian toxicity rhythm. The model has been derived using, as far as possible, experimental data. We examine two different optimisation problems. The eradication problem consists in finding the drug infusion law able to minimise the number of tumour cells while preserving a minimal level for the villi population. On the other hand, the containment problem searches for a quasi periodic treatment able to maintain the tumour population at the lowest possible level, while preserving the villi cells. The originality of these approaches is that the objective and constraint functions we use are $L^{\infty}$ criteria. We are able to derive their gradients with respect to the infusion rate and then to implement efficient optimisation algorithms.
\end{abstract}

Mathematics Subject Classification. 49M29, 92B05, 92C50.

Received: March 18, 2005.

\section{INTRODUCTION}

An important issue in the treatment of cancer is its tolerability by patients. Drugs that show good therapeutic effects by killing tumour cells are always limited in their use by their toxicity on healthy tissues. Such unwanted toxicity usually depends on the particular drug in use, but since they all act by hindering cell proliferation, the

\footnotetext{
Keywords and phrases. Dynamical systems, optimisation, circadian rhythms, drugs, therapeutics, cancer.

1 Université Paris-Nord, Villetaneuse and École Normale Supérieure, 75005 Paris, France. basdevant@lmd.ens.fr

2 INRIA, projet BANG, 78153 Rocquencourt, France. jean.clairambault@inria.fr (corresponding author)

3 INSERM E 0354, Hôpital Paul-Brousse, 94800 Villejuif, France. levi-f@vjf.inserm.fr
} 
most exposed healthy cell populations are found in normal fast renewing tissues, such as intestinal mucosa or bone marrow, where cell proliferation is as active as in tumour tissues - though under control.

It is thus of the greatest importance to find differences in the behaviours of healthy and cancer cells towards aggression by anti-tumour treatments. In this respect, to our knowledge, no clear molecular mechanisms have been documented as yet on these differences. But various observations at the macroscopic, cellular or molecular levels have been made and used to try and propose improved chemotherapy treatments in patients with cancer. In particular, according to these observations, the mean cell cycle time is often different between healthy and cancer cells, and even if it is the same, its variance should be higher in cancer cells, due to lack of synchronisation between cells in the cycle; this has led to propose pulsed chemotherapies of cell-cycle phase-specific drugs with the aim to destroy electively proliferating cancer cells $[1,9-11,34]$. More recently, it has been proposed that the ability to undergo apoptosis (programmed cell death) induced by cytotoxic drugs could be higher in tumour cells than in normal healthy cells, thus explaining the already observed good therapeutic index (anti-tumour efficacy vs. unwanted toxicity) of these drugs [14].

But another fact, which has also been observed for a rather long time at the experimental and clinical levels, is that a circadian rhythmicity of the pharmacosensitivity to these drugs (as for most drugs) exists, its phase depends on the particular drug in use, and it is different in cancer and in healthy cells [27]. A molecular basis supporting the influence of circadian clock genes on the expression of genes involved in cell cycle progression and apoptosis has recently been found $[16,17,28]$, and chronobiological research is at the present time very active on this topic, even if until now no molecular mechanism has been evidenced for circadian rhythm differences between normal and cancer cells.

The chronotherapy concept thus takes advantage of these observed circadian rhythmicity differences by maximising therapeutic efficacy on a tumour while minimising undesirable toxicity on healthy tissues [27]. One should point out that unlike pulsed therapies with an artificial external period, which may eventually present the drawback of resynchronising a desynchronised population of tumour cells according to this artificial period [2], circadian chronotherapy uses a natural period (circa $24 \mathrm{~h}$ ), which is present in healthy cells [22] and to a lesser extent in tumour cells [7], for pharmacological sensitivity, to deliver cytotoxic drugs to treat cancer. Furthermore, peripheral cells - and the subjects bearing them -, endowed with this common natural period, are also naturally phase-synchronised by inputs from the light-dark cycle ${ }^{1}$.

The object of the present paper is to investigate mathematically and numerically optimal strategies in (circadian) cancer chronotherapy. To this end a mathematical model describing the time evolution of efficacy and toxicity of intravenous oxaliplatin, one of the few drugs active on metastatic colorectal cancer, has been derived [8]. We have then applied an optimal control technique to obtain the best law for the drug infusion flow.

Many authors have previously addressed optimal control problems, often with $L^{1}$ or $L^{2}$ criteria, for anticancer chemotherapy. They did so mostly by taking into account acquired resistance of tumour cells to cytotoxic drugs, with or without cell cycle phase specificity (reviews in $[15,25,33]$ ), but usually without pharmacokineticpharmacodynamic (PK-PD) modelling. We address here neither drug resistance nor cell cycle phase specificity, but rather focus on a balance between anti-tumour efficacy and healthy tissue toxicity of cytotoxic drugs, as has also been done in $[23,24]$. In this limited setting, we consider our approach original inasmuch as it uses pure $L^{\infty}$ criteria (though using a nonlinear conjugate gradient method), includes PK-PD modelling, and takes into account drug circadian effects.

Section 2 will be devoted to the study of mathematical models for the cell populations submitted to drug infusion. We will first consider the healthy tissues and then the tumour cells. We will show that the minimal (or maximal) cell population during a given time interval happens to be a differentiable function of the drug infusion law, the gradient of which can be derived. In Section 3 two different optimisation problems are addressed; the eradication problem consists in finding the drug infusion law leading to the smallest, and possibly vanishing, tumour population; the containment problem looks for a treatment that forces the number of tumour cells to

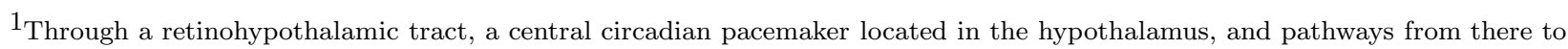
peripheral tissues still remaining to be elucidated, but which include the autonomic nervous system and corticoid hormones [27].
} 
remain at the lowest possible level while preserving an acceptable level for the healthy tissues. In Section 4 numerical results are presented; discussion and conclusion follow in Section 5.

\section{THE MODEL}

\subsection{Experimental and pharmacological background}

Given the scarcity of long followup human data on untreated tumour growth, we used animal experimentation. Oxaliplatin, one of the few drugs active on metastatic colorectal cancer, is also known to be active on Glasgow osteosarcoma in mice. This murine tumour, transplanted under the skin of the animal, is easily measurable at the laboratory with a caliper, which allows obtaining tumour growth curves, with or without treatment, in whole living animals[18] (animals in which tumours had reached 10\% of their total body weight were sacrificed for ethical reasons).

The main toxicities of oxaliplatin in Man are on bone marrow, peripheral sensory nerves, and the jejunum[26]. In mice, the main toxicity is on the jejunum (producing extended necrosis of the mucosa) [4,5], which led us to design a simple model for jejunal mucosa proliferation and homeostasis, which one may take as a paradigm of fast renewing healthy tissue behaviour subject to drug toxicity, within a global model designed to represent both therapeutic efficacy and toxicity.

Plasma pharmacokinetic parameters for oxaliplatin (a drug which binds covalently, i.e. irreversibly to its targets or to detoxicating molecules in living cells) have been taken from published data [4,5] and input to a simple first order model; tissue pharmacokinetic parameters (measuring the decrease of drug quantities in both types of tissues, tumour and jejunal mucosa) were identified either from published data[4,5], or by an overall estimation when data were not available. The efficacy or toxicity function describing the cell kill effect was chosen of the Hill type, taking into account pharmacological uses and recent published studies [13,30], its maximum being modulated by a $24 \mathrm{~h}$-periodic cosine representing circadian pharmacosensitivity. Tissue pharmacodynamic parameters - including maximal and minimal pharmacosensitivity phases - were identified as much as possible from laboratory curves[18] (comparison between treated and untreated tumour growth) or else only estimated on the basis of likeliness after other drug or tissue data when no relevant data were available.

\subsection{The healthy tissue: jejunal villi cells}

We chose for the representation of jejunal mucosa homeostasis a simple damped linear model, which mimics in a satisfactory way what is known of the recovery behaviour of enterocytes subject to radiotoxic or cytotoxic insult[31]. It may be thought of as the linearisation of a more complex nonlinear model[6] about a stable equilibrium point, which from a pathophysiological point of view is actually a very stable equilibrium point, representing tissue homeostasis. The drug toxicity effect was supposed to be active only on the compartment representing the influx of young cells to the jejunal villi, since mature cells are unable to proliferate in the jejunal mucosa, and oxaliplatin, as most anti-cancer drugs, is active mainly on proliferating cells.

\subsubsection{Mathematical model for villi population}

The mathematical model for the villi population $A(t)$ at time $t$ consists in a set of four coupled differential equations:

$$
\begin{aligned}
& \frac{\mathrm{d} P}{\mathrm{~d} t}=-\lambda P+\frac{i(t)}{V_{\mathrm{dist}}} \Phi(t) \\
& \frac{\mathrm{d} C}{\mathrm{~d} t}=-\mu C+\xi_{C} P \\
& \frac{\mathrm{d} Z}{\mathrm{~d} t}=\{-\alpha-f(C, t)\} Z-\beta A+\gamma \\
& \frac{\mathrm{d} A}{\mathrm{~d} t}=Z-Z_{e q} .
\end{aligned}
$$


Here $P$ and $C$ stand, respectively, for drug concentrations in the plasmatic and target cell population compartments (plasma and the jejunal mucosa, target of toxicity, i.e. of unwanted side effects of the drug). $V_{\text {dist }}$ is the distribution volume, i.e. the volume of the central (plasmatic) compartment, in which the active drug is infused. $A$ is the number of mature (villi) jejunal enterocytes, $Z$ is the instantaneous flow to villi from crypts, rate of renewal to make up for natural elimination of villi enterocytes in the intestinal lumen. $\Phi(t)$ is given, equal to 1 during authorized infusion periods and 0 elsewhere and $i(t) \geq 0$ is the time dependent drug infusion law. Function $f$ represents the drug toxicity on the healthy jejunal mucosa, assumed to be periodic, more precisely here to have circadian variations with period $T_{A}$; it is given by:

$$
f(C, t)=F\left(1+\cos \left(2 \pi \frac{t-\varphi_{A}}{T_{A}}\right)\right) \frac{C^{\gamma_{A}}}{C_{50}^{\gamma_{A}}+C^{\gamma_{A}}}
$$

$\lambda, \mu, \xi_{C}, \alpha, \beta, \gamma, Z_{e q}, F, \varphi_{A}, \gamma_{A}, C_{50}$ are positive constants. These equations represent drug diffusion and elimination by first order pharmacokinetics for concentrations in the plasmatic and target cell compartments $(P$ and $C)$, and normal jejunal mucosa homeostasis by a linear system showing a stable focus at $\left(Z_{e q}, A_{e q}=\beta^{-1}\left(\gamma-\alpha Z_{e q}\right)\right)$, perturbed by the drug toxicity function which comes to strengthen the natural self-regulation coefficient $\alpha$.

Classical ODE theorems demonstrate that, initial conditions at time $t_{0}$ been given for $X_{A}(t)=(P(t), C(t)$, $Z(t), A(t)$ ), and a piecewise continuous infusion profile $i(t)$ been prescribed, system (1-4) has a continuous, piecewise $C^{1}$, solution for $t \in\left[t_{0}, t_{f}\right]$ (for any $t_{f}>t_{0}$ ). Moreover:

$$
i \in L^{2}\left(\left[t_{0}, t_{f}\right]\right) \longrightarrow A \in H^{4}\left(\left[t_{0}, t_{f}\right]\right)
$$

is a continuous, weakly continuous, differentiable application, $A(t)$ being a $C^{3}$, piecewise $C^{4}$, function of time ${ }^{2}$.

Remark 1. Functions $P, C, Z, A$ and most functions in the following will be considered as functions of time $t$ and of $i$ the infusion law (which is also itself a function of time), however we will omit to note the $i$-dependency whenever there is no ambiguity, hence writing $A$ or $A(t)$ rather than $A(i, t)$.

Remark 2. Functions $P$ and $C$ are drug concentrations and thus positive quantities; equations (1) and (2) ensure they remain positive. Unfortunately equations (3)-(4) do not ensure the positivity of $A$, a population count; however, when $A(t)$ reaches zero the animal (or the patient: it is noteworthy that in clinical settings severe jejunal depletion may yield diarrhoeas which may be fatal) is already dead and the model is no longer valid!

In order to find optimal infusion laws by means of descent algorithms, we need to know the gradients with respect to the infusion law of objective or constraint functions, that is, if $W(i)$ is such a differentiable real function of $i \in L^{2}\left(\left[t_{0}, t_{f}\right]\right)$, and $\frac{\partial W}{\partial i}$ its differential with respect to $i$, the gradient $\nabla_{i} W(i)$ is the element of $L^{2}\left(\left[t_{0}, t_{f}\right]\right)$ such that:

$$
\frac{\partial W}{\partial i} \cdot d i=\left\langle\nabla_{i} W(i), d i\right\rangle
$$

${ }^{2}$ In fact, solution of (1) reads:

$$
P(t)=P\left(t_{0}\right) \mathrm{e}^{-\lambda t}+\int_{t_{0}}^{t} \frac{i(\tau)}{V} \Phi(\tau) \mathrm{e}^{-\lambda(t-\tau)} \mathrm{d} \tau,
$$

thus, if $i$ is in $L^{2}, P$ is in $H^{1}$ and the application from $L^{2}$ to $H^{1}$ is affine, continuous, weakly-continuous, differentiable. Moreover if $i$ is piecewise continuous, $P$ is continuous and piecewise $C^{1}$.

The behaviour of (2) is similar; if $P$ is $H^{1}$ then $C$ is an infusion profile piecewise continuous, $C$ is $C^{1}$ and piecewise $C^{2}$ in time. Now system (3-4) is linear with piecewise $C^{2}$ coefficients, thus system (3-4) has a solution that is $C^{2}$ and piecewise $C^{3}$, for a piecewise continuous infusion, therefore $A$ is $C^{3}$ and piecewise $C^{4}$. Function $f(C, t)$ being a Lispchitz function of $C, A$ is a regular function of $C$ and then a continuous, weakly continuous, differentiable application of $i$. 
where $\left\langle\nabla_{i} W(i), d i\right\rangle$ is the scalar product of $L^{2}\left(\left[t_{0}, t_{f}\right]\right)$ :

$$
\left\langle\nabla_{i} W(i), d i\right\rangle=\int_{t_{0}}^{t_{f}} \nabla_{i} W(i)(\tau) d i(\tau) \mathrm{d} \tau
$$

This will be done in the following paragraphs.

\subsubsection{A first function of the villi population}

Let us define, for $\eta$ a given time in $] t_{0}, t_{f}\left[\right.$, a function $\tilde{F}_{A}$ of the drug infusion law by

$$
\tilde{F}_{A}(i, \eta)=\tau_{A}-\frac{A(\eta)}{A_{e q}}
$$

where $A_{e q}$ is a reference level for the villi population and $\left.\left.\tau_{A} \in\right] 0,1\right]$ a tolerable fraction of this reference level. We know that $F_{A}$ is a continuous, weakly continuous, differentiable function of $i \in L^{2}\left(\left[t_{0}, t_{f}\right]\right)$. To obtain its gradient, we will demonstrate the following lemma:

Lemma 1. The gradient $\nabla_{i} \tilde{F}_{A}$ of $\tilde{F}_{A}$ with respect to the infusion profile $i$ is defined in $L^{2}\left(\left[t_{0}, t_{f}\right]\right)$ by:

$$
\nabla_{i} \tilde{F}_{A}= \begin{cases}\frac{\Phi(t)}{V_{\mathrm{dist}}} P_{a 1}(t) & \forall t \in\left[t_{0}, \eta\right] \\ 0 & \forall t>\eta\end{cases}
$$

where $P_{a 1}$ is given by the adjoint system, defined for $t_{0} \leq t \leq \eta$ :

$$
\begin{aligned}
\frac{\mathrm{d} P_{a 1}}{\mathrm{~d} t} & =\lambda P_{a 1}-\xi_{C} P_{a 2} \\
\frac{\mathrm{d} P_{a 2}}{\mathrm{~d} t} & =\mu P_{a 2}+P_{a 3} Z \frac{\partial f}{\partial C}(C, t) \\
\frac{\mathrm{d} P_{a 3}}{\mathrm{~d} t} & =\{\alpha+f(C, t)\} P_{a 3}-P_{a 4} \\
\frac{\mathrm{d} P_{a 4}}{\mathrm{~d} t} & =\beta P_{a 3}
\end{aligned}
$$

with initial conditions at time $\eta$ :

$$
P_{a 1}(\eta)=P_{a 2}(\eta)=P_{a 3}(\eta)=0 \quad \text { and } \quad P_{a 4}(\eta)=-\frac{1}{A_{e q}}
$$

The proof of the lemma can be obtained by mere identification. Indeed, one has to verify that for any $d i \in$ $L^{2}\left(\left[t_{0}, t_{f}\right]\right)$ :

$$
\frac{\partial \tilde{F}_{A}}{\partial i} \cdot d i=-\frac{\bar{A}(\eta)}{A_{e q}}=\int_{t_{0}}^{t_{f}} \nabla_{i} \tilde{F}_{A}(\tau) d i(\tau) \mathrm{d} \tau
$$


where $\bar{A}=\frac{\partial A}{\partial i} \cdot d i$, the differential of $A$ with respect to $i$ applied to $d i$, is defined by the following linear tangent system, defined for $t \geq t_{0}$ :

$$
\begin{aligned}
& \frac{\mathrm{d} \bar{P}}{\mathrm{~d} t}=-\lambda \bar{P}+\frac{d i(t)}{V_{\mathrm{dist}}} \Phi(t) \\
& \frac{\mathrm{d} \bar{C}}{\mathrm{~d} t}=-\mu \bar{C}+\xi_{C} \bar{P} \\
& \frac{\mathrm{d} \bar{Z}}{\mathrm{~d} t}=\{-\alpha-f(C, t)\} \bar{Z}-\frac{\partial f}{\partial C}(C, t) \bar{C} Z-\beta \bar{A} \\
& \frac{\mathrm{d} \bar{A}}{\mathrm{~d} t}=\bar{Z}
\end{aligned}
$$

with vanishing initial conditions at $t=t_{0}$.

The calculation then goes as follows; if $\nabla_{i} \tilde{F}_{A}$ is defined by (5), then:

$$
\begin{aligned}
\int_{t_{0}}^{t_{f}} \nabla_{i} \tilde{F}_{A}(\tau) d i(\tau) \mathrm{d} t & =\int_{t_{0}}^{\eta} \nabla_{i} \tilde{F}_{A}(\tau) d i(\tau) \mathrm{d} \tau \\
& =\int_{t_{0}}^{\eta} P_{a 1}(\tau) \frac{\Phi(\tau)}{V_{\text {dist }}} d i(\tau) \mathrm{d} \tau
\end{aligned}
$$

and using equations (11)-(14):

$$
\begin{aligned}
= & \int_{t_{0}}^{\eta}\left\{P_{a 1}(\tau)\left(\frac{\mathrm{d} \bar{P}}{\mathrm{~d} t}+\lambda \bar{P}\right)+P_{a 2}(\tau)\left(\frac{\mathrm{d} \bar{C}}{d t}+\mu \bar{C}-\xi_{C} \bar{P}\right)\right. \\
& +P_{a 3}(\tau)\left(\frac{\mathrm{d} \bar{Z}}{\mathrm{~d} t}+\{\alpha+f(C, t)\} \bar{Z}+\frac{\partial f}{\partial C}(C, t) \bar{C} Z+\beta \bar{A}\right) \\
& \left.+P_{a 4}(\tau)\left(\frac{\mathrm{d} \bar{A}}{\mathrm{~d} t}-\bar{Z}\right)\right\} \mathrm{d} \tau
\end{aligned}
$$

doing one integration by parts, the result (10) is obtained using equations (6)-(9) and the boundary conditions at $t_{0}$ for the $\bar{P}, \bar{C}, \bar{Z}, \bar{A}$ and at $\eta$ for $P_{a 1}, P_{a 2}, P_{a 3}, P_{a 4}$.

\subsubsection{The minimal villi population}

Now consider the constraint:

$$
F_{A}(i)=\tau_{A}-\min _{t \in\left[t_{0}, t_{f}\right]} \frac{A(t)}{A_{e q}} \leq 0,
$$

where again $A_{e q}$ is a reference level for the villi population and $\left.\left.\tau_{A} \in\right] 0,1\right]$ a tolerable fraction of this reference level. We know that $F_{A}$ is a continuous, weakly continuous, real function of $i \in L^{2}\left(\left[t_{0}, t_{f}\right]\right)$. The following theorem tells us that in most cases the gradient of $F_{A}$ exists and can be computed using the adjoint system (6)-(9).

Theorem 1. If in the vicinity of infusion $i$ the minimum of $A(t)$ is unique, belongs to $] t_{0}, t_{f}$ [ with a strictly positive second derivative, then $F_{A}$ is a differentiable function of $i$. If we denote by $t_{A}(i)$ the time at which $A$ reaches its minimum, then the gradient $\nabla_{i} F_{A}$ is given by:

$$
\nabla_{i} F_{A}(i)(t)= \begin{cases}\nabla_{i} \tilde{F}_{A}\left(i, t_{A}\right)(t) & \forall t \in\left[t_{0}, t_{A}\right] \\ 0 & \forall t>t_{A}\end{cases}
$$

Let us show first that, under these hypothesis, $t_{A}$ is a differentiable function of $i$. In fact $t_{A}$ is locally in time defined by the equation $\frac{\partial A\left(i, t_{A}(i)\right)}{\partial t}=0$. With the hypothesis that $\frac{\partial^{2} A\left(i, t_{A}(i)\right)}{\partial t^{2}}>0$, and the property that $A$ is 
a differentiable function of $i$, the implicit function theorem applied to the equation $\frac{\partial A(i, t)}{\partial t}=0$ gives the result. Then the computation of the gradient relies on the differentiation chain rule: as $F_{A}(i)=\tilde{F}_{A}\left(i, t_{A}\right)$,

$$
\frac{\partial F_{A}(i)}{\partial i} \cdot d i=\frac{\partial \tilde{F}_{A}\left(i, t_{A}\right)}{\partial i} \cdot d i+\frac{\partial \tilde{F}_{A}\left(i, t_{A}\right)}{\partial t} \frac{\partial t_{A}}{\partial i} \cdot d i .
$$

The minimum of $A(t)$ been reached at time $t_{A}$ it follows that $\frac{\partial \tilde{F}_{A}\left(i, t_{A}\right)}{\partial t}=0$ and the results follows.

\subsection{The tumour cell population}

Tumour growth was modelled according to a Gompertz law, which is one of the simplest laws classically used for this purpose $[12,20,21]$. The variable $B$ which stands for tumour cell population number (see equations below) is in this model bound to eventually reach a plateau $B_{\max }$, after an initial exponential growth, following an S-shaped curve. The accuracy of the Gompertz model for tumour growth has often been questioned, but also justified on refined modelling grounds (using quiescent and proliferative subpopulations inside the tumour); it is generally accepted at least as a first intention simple modelling approach for this purpose, and we decided to use it in this sense.

\subsubsection{Mathematical model for the tumour cell population}

The mathematical model for the tumour cell population $B(t)$ at time $t$ consists in a set of three coupled differential equations:

$$
\begin{aligned}
\frac{\mathrm{d} P}{\mathrm{~d} t} & =-\lambda P+\frac{i(t)}{V_{\mathrm{dist}}} \Phi(t) \\
\frac{\mathrm{d} D}{\mathrm{~d} t} & =-\nu D+\xi_{D} P \\
\frac{\mathrm{d} B}{\mathrm{~d} t} & =-a B \ln \left(\frac{B}{B_{\max }}\right)-g(D, t) B
\end{aligned}
$$

where equation (15) is the same as (1), D stands for drug concentration (assumed to be homogeneously diffused) in the tumour and $B$ is the number of tumour cells. Function $g$, which represents anti-tumour drug efficacy, is assumed (as is function $f$ for toxicity) to present circadian variations with period $T_{B}$; it is given by:

$$
g(D, t)=H\left(1+\cos \left(2 \pi \frac{t-\varphi_{B}}{T_{B}}\right)\right) \frac{D^{\gamma_{B}}}{D_{50}^{\gamma_{B}}+D^{\gamma_{B}}}
$$

where $\lambda, \nu, \xi_{D}, a, B_{\max }, H, \varphi_{B}, \gamma_{B}, D_{50}$ are positive constants.

Initial conditions at time $t_{0}$ been given for $X_{B}(t)=(P(t), D(t), B(t))$, and a piecewise continuous infusion profile $i(t)$ been prescribed, system (15)-(17) has a continuous, piecewise $C^{1}$, solution for $t \in\left[t_{0}, t_{f}\right]$ for any $t_{f}>t_{0}$. Moreover:

$$
i \in L^{2}\left(\left[t_{0}, t_{f}\right]\right) \longrightarrow B \in H^{3}\left(\left[t_{0}, t_{f}\right]\right)
$$

is a continuous, weakly continuous, differentiable application, $B(t)$ being a $C^{2}$, piecewise $C^{3}$, function of time. The demonstration is similar to the one for $A$ (note that Eq. (17) is a linear equation for the unknown $\ln (B)$ ).

Remark 3. Equations (15)-(17) ensure the positivity of $P, C$ and $B$. Nevertheless we will consider that the tumour has been completely destroyed if $B(t)<1$, thus integration of equation (17) has to be stopped when this arises.

Equation (17) is made of the Gompertz law for the tumour cell population growth and of a linear destruction law (modulated by the circadian effect) for the anti-tumour efficacy. The latter has at least two drawbacks. 
First, the drug is less and less efficient when the tumour gets smaller, one may wonder if this behaviour is realistic. Conversely, the drug is more and more efficient when the tumour gets larger. These two extreme behaviours explain why the numerical results are in practice nearly independent of the initial size $B(0)$ of the tumour. An alternative modelling assumption could be to replace $-g(D, t) B$ by a non linear term such as $-g(D, t) \frac{B}{1+B}$ in equation (17). But in the absence of experimental or clinical data supporting this hypothesis, we decided to keep the growth inhibition term in its linear form.

\subsubsection{A first function of the tumour cell population}

Let us define, for $\eta \in\left[t_{0}, t_{f}\right]$, a function $\tilde{F}_{B}$ of the drug infusion law by:

$$
\tilde{F}_{B}(i, \eta)=B(\eta)
$$

We know that $F_{B}$ is a continuous, weakly continuous, differentiable function of $i \in L^{2}\left(\left[t_{0}, t_{f}\right]\right)$. We will demonstrate the following lemma:

Lemma 2. The gradient $\nabla_{i} \tilde{F}_{B}$ of $\tilde{F}_{B}$ with respect to the infusion profile $i$ is defined in $L^{2}\left(\left[t_{0}, t_{f}\right]\right)$ by:

$$
\nabla_{i} \tilde{F}_{B}= \begin{cases}\frac{\Phi(t)}{V_{\text {dist }}} P_{b 1}(t) & \forall t \in\left[t_{0}, \eta\right] \\ 0 & \forall t>\eta\end{cases}
$$

where $P_{b 1}$ is given by the adjoint system, defined for $t_{0} \leq t \leq \eta$ :

$$
\begin{aligned}
& \frac{\mathrm{d} P_{b 1}}{\mathrm{~d} t}=\lambda P_{b 1}-\xi_{D} P_{b 2} \\
& \frac{\mathrm{d} P_{b 2}}{\mathrm{~d} t}=\nu P_{b 2}+P_{b 3} B \frac{\partial g}{\partial D}(D, t) \\
& \frac{\mathrm{d} P_{b 3}}{\mathrm{~d} t}=P_{b 3}\left(a \ln \left(\frac{B}{B_{\max }}\right)+a-g(D, t)\right)
\end{aligned}
$$

with initial conditions at time $\eta$ :

$$
P_{b 1}(\eta)=P_{b 2}(\eta)=0 \quad \text { and } \quad P_{b 3}(\eta)=1
$$

As it has been done for $\tilde{F}_{A}$, the proof of the lemma can be obtained by mere identification. For any $d i \in$ $L^{2}\left(\left[t_{0}, t_{f}\right]\right)$ :

$$
\frac{\partial \tilde{F}_{B}}{\partial i} . d i=\bar{B}(\eta)=\int_{t_{0}}^{t_{f}} \nabla_{i} \tilde{F}_{B}(\tau) d i(\tau) \mathrm{d} \tau
$$

where $\bar{B}=\frac{\partial B}{\partial i}$.di, the differential of $B$ with respect to $i$ applied to $d i$, is obtained by solving the following linear tangent system, defined for $t \geq t_{0}$ :

$$
\begin{aligned}
\frac{\mathrm{d} \bar{P}}{\mathrm{~d} t} & =-\lambda \bar{P}+\frac{d i(t)}{V_{\mathrm{dist}}} \Phi(t) \\
\frac{\mathrm{d} \bar{D}}{\mathrm{~d} t} & =-\nu \bar{D}+\xi_{D} \bar{P} \\
\frac{\mathrm{d} \bar{B}}{\mathrm{~d} t} & =-a \bar{B} \ln \left(\frac{B}{B_{\max }}\right)-a \bar{B}-g(D, t) \bar{B}-\frac{\partial g}{\partial D}(D, t) \bar{D} B
\end{aligned}
$$

with vanishing initial conditions at $t=t_{0}$. 


\subsubsection{The maximal (or minimal) tumour cell population}

Now consider the objective function:

$$
J_{B}(i)=\max _{t \in\left[t_{1}, t_{f}\right]} B(t),
$$

where $t_{1}$ is a given time after the initial phase of the treatment. We know that $J_{B}$ is a continuous, weakly continuous, real function of $i \in L^{2}\left(\left[t_{0}, t_{f}\right]\right)$. The following theorem tells us that in most cases the gradient of $J_{B}$ exists and can be computed using the adjoint system (18)-(20).

Theorem 2. If in the vicinity of infusion $i$ the maximum of $B(t)$ in $] t_{1}, t_{f}$ [ is unique with a strictly negative second derivative, then $J_{B}$ is a differentiable function of $i$. If we denote by $t_{B}(i)$ the time at which $B$ reaches its maximum, then the gradient $\nabla_{i} J_{B}$ is given by:

$$
\nabla_{i} J_{B}(i)(t)= \begin{cases}\nabla_{i} \tilde{F}_{B}\left(i, t_{B}\right)(t) & \forall t \in\left[t_{0}, t_{B}\right] \\ 0 & \forall t>t_{B}\end{cases}
$$

The proof of the theorem is similar to the one for the villi population and relies on the differentiation chain rule as $J_{B}(i)=\tilde{F}_{B}\left(i, t_{B}\right)$.

Remark 4. A similar result is valid for $G_{B}(i)=\min _{t \in\left[t_{0}, t_{f}\right]} B(t)$.

\section{Two Optimisation PROBlems}

\subsection{The eradication problem}

Let us consider the problem of finding the infusion law leading to the smallest, and possibly vanishing, tumour population while preserving a minimal villi population. Mathematically this can be written: find the infusion law $i \in L^{2}\left(\left[t_{0}, t_{f}\right]\right), i(t) \geq 0$, that minimises the objective function:

$$
G_{B}(i)=\min _{t \in\left[t_{0}, t_{f}\right]} B(t)
$$

subject to the constraint

$$
F_{A}(i)=\tau_{A}-\min _{t \in\left[t_{0}, t_{f}\right]} \frac{A(t)}{A_{e q}} \leq 0 .
$$

As $F_{A}$ is weakly continuous, the ensemble of admissible solutions

$$
U=\left\{i \in L^{2}\left(\left[t_{0}, t_{f}\right]\right) \mid 0 \leq i, F_{A} \leq 0\right\}
$$

is weakly closed in $L^{2}\left(\left[t_{0}, t_{f}\right]\right)$; as the infused drug destroys the villi population $U$ is also bounded; $G_{B}$ being weakly continuous, this ensures the existence of an optimal infusion law for the eradication problem. However neither the objective function, nor the constraint are convex functions of the infusion law. The optimum may not be unique (indeed $i(t)$ remains undefined where $\Phi(t)=0$ ) and, moreover, there may exist local minima. Nevertheless the results from previous sections allow us to define descent algorithms to find quasi-optimal strategies. A Uzawa-like saddle-point algorithm (see e.g. [3]) for solving the eradication problem is:

(1) Start with a Lagrange multiplier $\alpha_{A}^{0}>0$.

(2) Find $i^{k}$, minimising $J(i)=G_{B}(i)+\alpha_{A}^{k} F_{A}(i)$ in $L^{2}\left(\left[t_{0}, t_{f}\right]\right) \cap\{i \geq 0\}$.

(3) Define $\alpha_{A}^{k+1}$ by $\alpha_{A}^{k+1}=\max \left\{\alpha_{A}^{k}+\rho F_{A}\left(i^{k}\right), 0\right\}$.

(4) Until $F_{A}\left(i^{k}\right) \approx 0$.

In step (3) the coefficient $\rho>0$ has to be chosen adequately (in fact this step corresponds to a gradient algorithm for the dual problem). In practice we found more efficient to use for step (3) a bisection or a secant algorithm to find the Lagrange multiplier $\alpha_{A}$ associated to the active constraint $F_{A}=0$. 
Remark 5. If other constraints are to be imposed on the infusion law, such for example a bound for the total infusion or a maximum instantaneous infusion rate, these constraints can be treated either in step (2) or using more Lagrange multipliers.

\subsection{The containment problem}

Unfortunately there may not exist a treatment that destroys down to a sufficiently low level the tumour cells while keeping a sufficiently high level of villi cells. As the number of villi cells is in clinical settings of primary importance for the patient's life, one has then to reduce the infusion rate and consequently the treatment course will end with a non negligible number of residual tumour cells. Due to the Gompertz law, the tumour will then grow up rapidly after the end of the course. In that context one has to look for a containment treatment, that is a treatment repeated periodically in time that forces the number of tumour cells to remain at the lowest possible level while preserving an acceptable number of villi cells. Mathematically this can be written: find the infusion law $i \in L^{2}\left(\left[t_{0}, t_{f}\right]\right), i(t) \geq 0$, that minimises the objective function:

$$
J_{B}(i)=\max _{t \in\left[t_{1}, t_{f}\right]} B(t)
$$

subject to the constraint

$$
F_{A}(i)=\tau_{A}-\min _{t \in\left[t_{0}, t_{f}\right]} \frac{A(t)}{A_{e q}} \leq 0 .
$$

In that case function $\Phi(t)$, that defines infusion periods, should for example take the value 1 during two days, then 0 during five days (no treatment), and then repeatedly until $t_{f}$; as for $t_{1}$ it can be taken to be 1 or 2 days after $t_{0}$ in order to allow the initial infusion to reduce significantly the tumor.

In the same way as for the eradication problem, $J_{B}$ is a weakly continuous function on a bounded weakly closed ensemble, thus the optimal infusion exists, however the minimum may not be unique and local minima may also exist.

The Uzawa-like algorithm defined previously can be applied similarly to the containment problem:

(1) Start with a Lagrange multiplier $\alpha_{A}^{0}>0$.

(2) Find $i^{k}$, minimising $J(i)=J_{B}(i)+\alpha_{A}^{k} F_{A}(i)$ in $L^{2}\left(\left[t_{0}, t_{f}\right]\right) \cap\{i \geq 0\}$.

(3) Define $\alpha_{A}^{k+1}$ by $\alpha_{A}^{k+1}=\max \left\{\alpha_{A}^{k}+\rho F_{A}\left(i^{k}\right), 0\right\}$.

(4) Until $F_{A}\left(i^{k}\right) \approx 0$.

\subsection{A descent algorithm}

We will briefly describe a descent algorithm designed to solve the minimisation problem in step (2) of the Uzawa procedure for the containment problem (or the eradication problem).

(1) Start from an initial infusion profile $i_{0}$.

(2) Given the infusion profile $i_{k}$, integrate the dynamical system (1)-(4) and (16)-(17) from $t_{0}$ until $t_{f}$ to obtain villi and tumour populations.

(3) Search for $t_{A}$ and $t_{B}$ and compute the value of $J_{B}, F_{A}$ and $J$. (Note: if accidentally an extremum value is reached at multiple times, choose the largest).

(4) Integrate the adjoint system (6)-(9) of $F_{A}$ from $t_{A}$ down to $t_{0}$ and the adjoint system $(18)-(20)$ of $J_{B}$ from $t_{B}$ down to $t_{0}$, to obtain the gradient of $J$.

(5) Determine a descent direction $d_{k}$ using the gradient of $J$ and previous descent directions (non-linear conjugate gradient, see $e . g .[3]))$.

(6) Determine $i_{k+1}$ by minimising $J\left(i_{k}+s d_{k}\right)$ along direction $d_{k}$ (one-dimensional search) under the constraint $i \geq 0$.

(7) Loop to step 2 until convergence. 
Remark 6. The one-dimensional search of step (6) asks for one or several evaluations of $J$ and eventually its gradient (Wolfe's search, see e.g. [3]), that is integrations of the dynamical system and of the adjoint systems.

Remark 7. During the one-dimensional search of step (6), several infusion profiles are tested with respect to the minimum of $J$. But incidentally one of them may be a good solution for the containment problem. It is then worthwhile (and costless) to compare, on the fly, with respect to $F_{A}$ and $J_{B}$, these infusion profiles to the best profile already encountered.

Remark 8. It is in practice efficient to take for $i_{0}$ in step 1 of the descent algorithm a combination of $i^{(k-1)}$, the last obtained infusion in the Uzawa procedure, and a constant infusion rate. Also a small random perturbation may be applied to $i_{0}$ in order to improve the exploration of the phase space.

\subsection{Optimisation with respect to the infusion period}

Let us suppose that the authorised infusion period is a single interval, thus $\Phi(t)=\chi_{\left[t_{0}, t_{i}\right]}(t)$. Then, in the eradication problem, the objective function $G_{B}$ and the constraint $F_{A}$ can be considered as functions of $i$ and of $t_{i}$; the optimisation can be conducted on the product space $L^{2}\left(\left[t_{0}, t_{f}\right]\right) \times\left[t_{0}, t_{f}\right]$. The only difference with the previous developments is that we need to compute the gradient of the various functions with respect to $t_{i}$ (gradients are in fact simple derivatives in this case). These are, for $F_{A}\left(i, t_{i}\right)=\tau_{A}-\min _{t \in\left[t_{0}, t_{f}\right]} \frac{A(t)}{A_{e q}}$ :

$$
\nabla_{t_{i}} F_{A}\left(i, t_{i}\right)= \begin{cases}-\frac{\check{A}\left(t_{A}\right)}{A_{e q}} & \text { if } t_{i}<t_{A} \\ 0 & \text { if not }\end{cases}
$$

with $A\left(t_{A}\right)=\min _{\left[t_{0}, t_{f}\right]} A(t)$, and $\breve{A}$ given by the linear tangent system, defined for $t \geq t_{i}$ :

$$
\begin{aligned}
& \frac{\mathrm{d} \check{P}}{\mathrm{~d} t}=-\lambda \check{P}+\frac{i(t)}{V_{\mathrm{dist}}} \delta\left(t-t_{i}\right) \\
& \frac{\mathrm{d} \check{C}}{\mathrm{~d} t}=-\mu \check{C}+\xi_{C} \check{P} \\
& \frac{\mathrm{d} \check{Z}}{\mathrm{~d} t}=\{-\alpha-f(C, t)\} \check{Z}-\frac{\partial f}{\partial C}(C, t) \check{C} Z-\beta \check{A} \\
& \frac{\mathrm{d} \check{A}}{\mathrm{~d} t}=\check{Z}
\end{aligned}
$$

where $\delta(t)$ is the Dirac function at origin and with vanishing initial conditions at $t=t_{i}$.

And for $G_{B}\left(i, t_{i}\right)=\min _{t \in\left[t_{0}, t_{f}\right]} B(t)$ :

$$
\nabla_{t_{i}} G_{B}\left(i, t_{i}\right)= \begin{cases}\check{B}\left(t_{B}\right) & \text { if } t_{i}<t_{B} \\ 0 & \text { if not }\end{cases}
$$


with $B\left(t_{B}\right)=\min _{\left[t_{0}, t_{f}\right]} B(t)$, and $\check{B}$ given by the linear tangent system, defined for $t \geq t_{i}$ :

$$
\begin{aligned}
\frac{\mathrm{d} \check{P}}{\mathrm{~d} t} & =-\lambda \check{P}+\frac{i(t)}{V_{\mathrm{dist}}} \delta\left(t-t_{i}\right) \\
\frac{\mathrm{d} \check{D}}{\mathrm{~d} t} & =-\nu \check{D}+\xi_{D} \check{P} \\
\frac{\mathrm{d} \check{B}}{\mathrm{~d} t} & =-a \check{B} \ln \left(\frac{B}{B_{\max }}\right)-a \check{B}-g(D, t) \check{B}-\frac{\partial g}{\partial D}(D, t) \check{D} B
\end{aligned}
$$

with vanishing initial conditions at $t=t_{i}$.

\section{NUMERICAL EXPERIMENTS}

We conducted several numerical experiments for both the containment and eradication problems. These experiments used the following data:

- For drug infusion: $\lambda=6 \mathrm{~h}^{-1} ; V_{\mathrm{dist}}=10 \mathrm{~cm}^{3}$ (distribution volume for oxaliplatin in a mouse).

- For the villi population:

$$
\begin{aligned}
& \mu=0.015 \mathrm{~h}^{-1}, \xi_{C}=1 \mathrm{~h}^{-1}, A_{e q}=10^{6} \text { cells, } Z_{e q}=16500 \text { cells } / \mathrm{h}, \alpha=0.0153 \mathrm{~h}^{-1}, \beta^{3}=0.002 \mathrm{~h}^{-2} \\
& \gamma=\beta A_{e q}+\alpha Z_{e q}=2213.8 \text { cells } / \mathrm{h}, F=0.5 \mathrm{~h}^{-1}, \varphi_{A}=10 \mathrm{~h}, T_{A}=24 \mathrm{~h}, \gamma_{A}=1, C_{50}=10 \mu \mathrm{g} / \mathrm{cm}^{3} .
\end{aligned}
$$

- For the tumour population:

$$
\begin{aligned}
& \nu=0.03 \mathrm{~h}^{-1}, \xi_{D}=1 \mathrm{~h}^{-1}, a=0.015 \mathrm{~h}^{-1}, B_{\max }=5.310^{6} \text { cells, } \\
& H=2 \mathrm{~h}^{-1}, \varphi_{B}=21 \mathrm{~h}, T_{B}=24 \mathrm{~h}, \gamma_{B}=1, D_{50}=10 \mu \mathrm{g} / \mathrm{cm}^{3} .
\end{aligned}
$$

These numerical data have been deduced from laboratory experiments; however, a large uncertainty remains on most of them, thus the goal of the subsequent numerical results is only to prove the feasibility of our approach rather than to obtain clinically relevant results.

All the experiments start with initial conditions: $P_{0}=C_{0}=D_{0}=0 \mu \mathrm{g} / \mathrm{cm}^{3}, A_{0}=10^{6}$ cells, $B_{0}=$ $10^{6}$ cells, $Z_{0}=16500$ cells $/ \mathrm{h}$. The initial time $t_{0}$ was noon and the time step 0.1 hour.

\subsection{Eradication problem}

The results presented were obtained in the framework of the eradication problem when optimising with respect both to $i$, the injection rate, and $t_{i}$, the infusion period. Together with the constraint to preserve a given level $\tau_{A}$ of the villi population, a constraint was also imposed on the instantaneous infusion rate, namely $i(t) \leq 10 \mu \mathrm{g} / \mathrm{h}^{4}$. Experiments started with a treatment duration of 48 hours ( $t_{i}-t_{0}=2$ days), and they converged to a treatment of almost 32 hours.

Figures 1 to 3 display respectively, as a function of time, the best infusion rate $i(t)$, the villi population $A(t)$ and the tumour cell population $B(t)$ for three different values of the minimal admissible fraction of the villi population $\tau_{A}=0.4,0.5$ and 0.6 . In Figure 1 , the impact of the different circadian rhythms for the villi and tumour populations is clearly seen as they impose a strong chronomodulation on the infusion rate. This impact is enhanced when the constraint to preserve the villi population is stronger. Note also the effect of the limitation of the instantaneous infusion rate. The chronomodulation is also seen on the villi population behaviour displayed in Figure 2.

\footnotetext{
${ }^{3} \alpha=\frac{1}{72} \ln 3, \beta=\left(\frac{\alpha}{2}\right)^{2}+\left(\frac{2 \pi}{144}\right)^{2}$, these values correspond to coefficients of a damped harmonic oscillator of period 6 days and dampening coefficient $\frac{1}{3}$ over one period, a behaviour estimated after literature data.

${ }^{4}$ The ensemble of admissible solutions is then $U=\left\{i \in L^{2}\left(\left[t_{0}, t_{f}\right]\right) \mid 0 \leq i \leq 10 \mu \mathrm{g} / \mathrm{h}, F_{A} \leq 0\right\}$; the maximal constraint on infusion rate is treated in step 2 of the algorithm (see Rem. 6).
} 


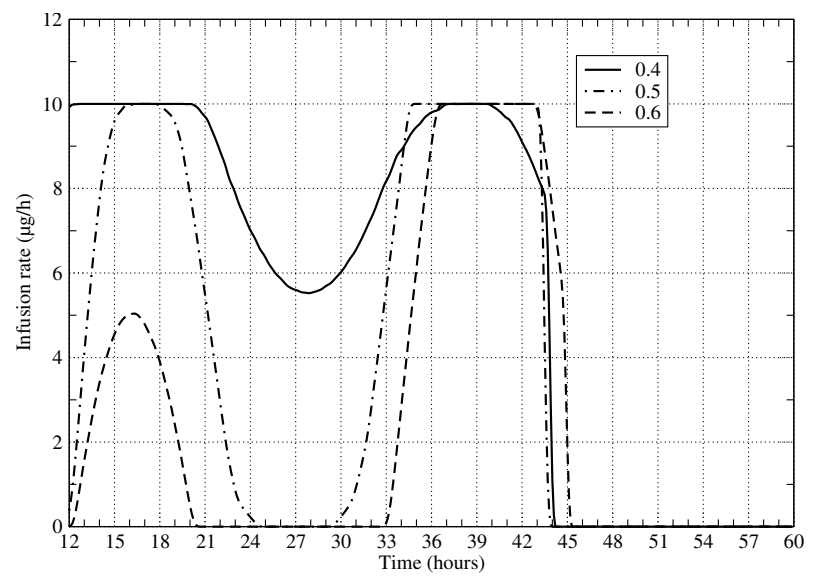

FIgURE 1. One week eradication treatment with $1.5+5.5$ days cycle: optimised infusion flows for $\tau_{A}=0.4,0.5$ and 0.6 .

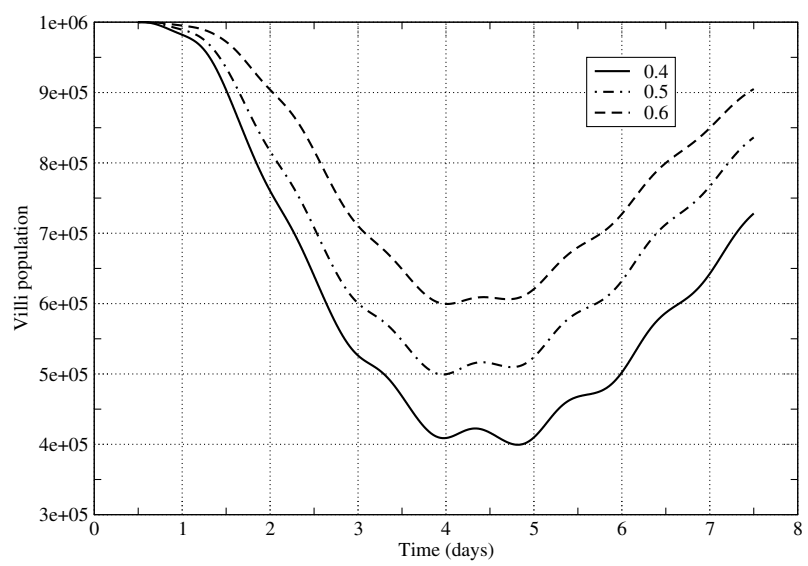

FIGURE 2. One week eradication treatment with $1.5+5.5$ days cycle: villi population for $\tau_{A}=0.4,0.5$ and 0.6 .

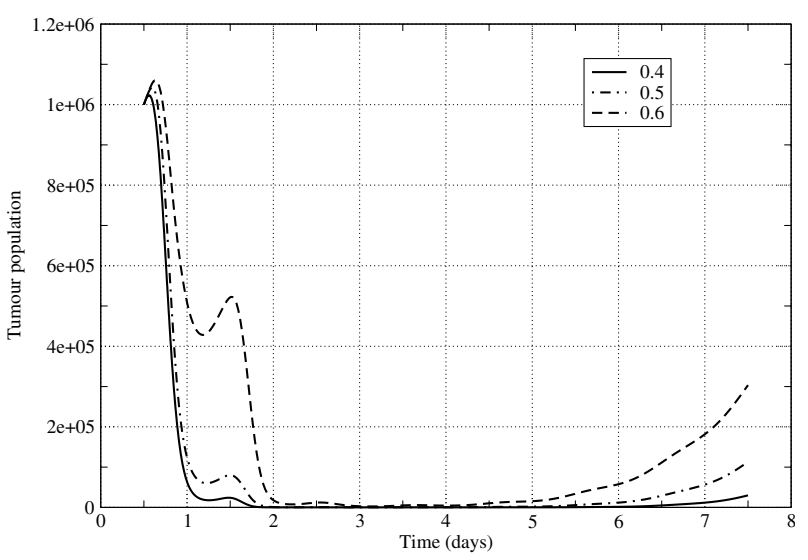

FiguRE 3. One week eradication treatment with $1.5+5.5$ days cycle: tumour cell population for $\tau_{A}=0.4$, 0.5 and 0.6 .

TABLE 1. Treatment durations and residual tumour cell numbers (eradication).

\begin{tabular}{cccc}
\hline & (days) & (hours) & \\
$\tau_{A}$ & $t_{i}-t_{0}$ & $t_{i}-t_{0}$ & $\min _{t} B(t)$ \\
\hline 0.4 & 1.33 & 31.9 & 3.4 \\
0.5 & 1.32 & 31.7 & 114 \\
0.6 & 1.38 & 34 & 2374 \\
\hline
\end{tabular}

Table 1 presents the best treatment duration $t_{i}-t_{0}$ and objective function $\min _{t} B(t)$ for the best infusion rate $i$, for the three experiments. The tumour population is strongly reduced when ${ }^{t} \tau_{A}$ decreases, however, with the data we used, the complete eradication of the tumour $(B<1)$ would require to diminish the villi population lower than $39 \%$ of the reference number, whatever the duration of the treatment, a level which, in a clinical context, is not admissible for the patient. Thus, for the experiments presented, a few tumour cells remain after 


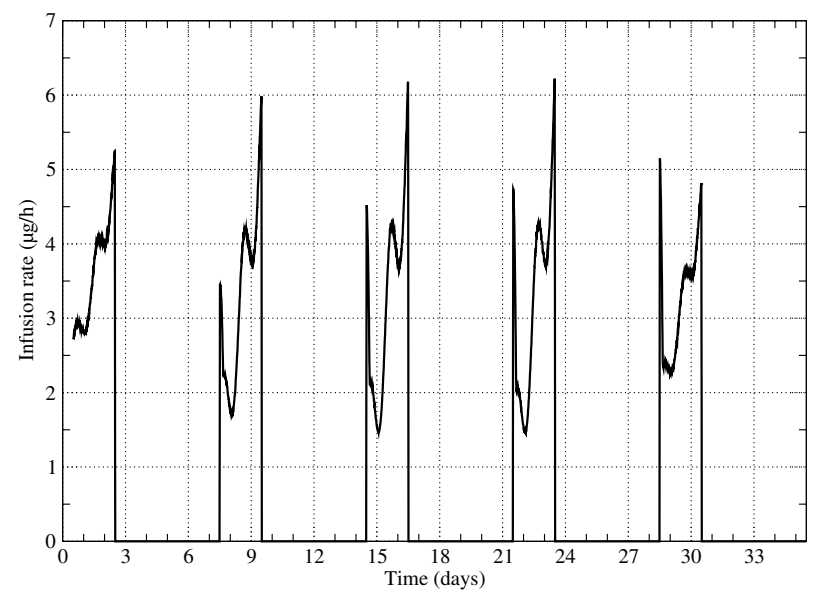

FIGURE 4. Five weeks containment treatment with $2+5$ days cycle: optimised drug infusion flow for $\tau_{A}=0.5$.

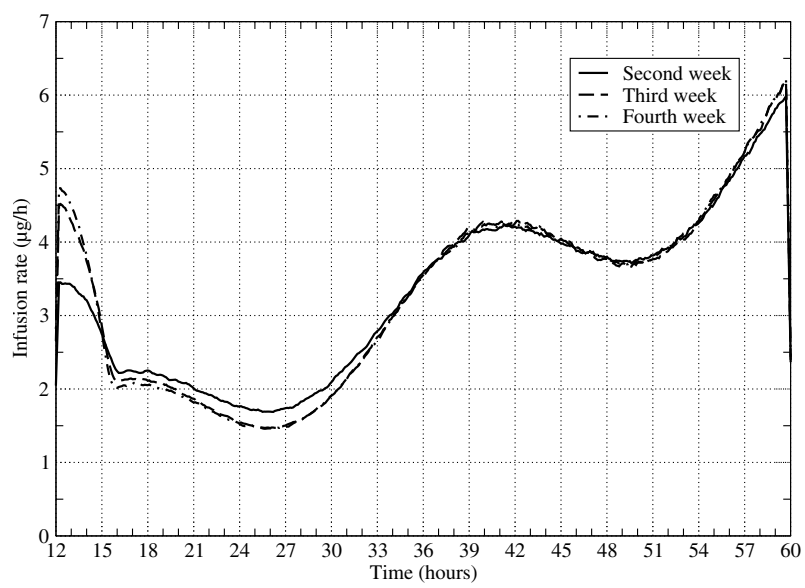

FiguRE 5. Five weeks containment treatment with $2+5$ days cycle: optimised drug infusion flows for $\tau_{A}=$ 0.5 , according to the week of treatment.

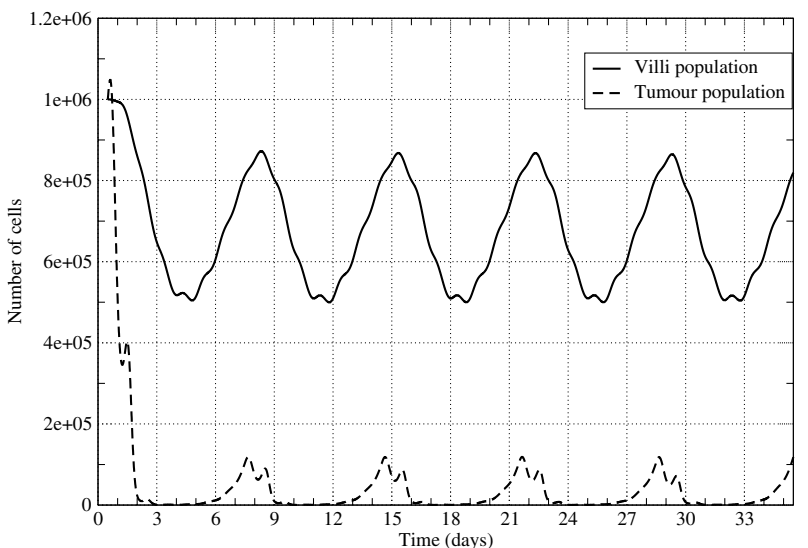

FiguRE 6. Five weeks containment treatment with $2+5$ days cycle: cell populations for $\tau_{A}=0.5$.

the end of the treatment (see Fig. 3), and, due to its exponential growth the tumour population will, without treatment, rapidly recover and overtake its initial level. This is why we studied the containment problem which should be applied to control tumour growth when the complete eradication is out of reach.

\subsection{Containment problem}

Figures 4 to 6 present the results for the containment problem with a weekly scenario of an infusion period of 2 days followed by 5 days of recovery, and an acceptable fraction $\tau_{A}=0.5$ for the villi population. The calculation was performed on a five weeks time interval. Figure 4 presents the infusion law, some details of which are shown in Figure 5. The infusion law is, as expected, strongly chronomodulated. Also, after the initial decrease of the tumour population, the infusion law evolves toward a periodic profile; indeed in Figure 5, curves become quickly intertwined. The tumour and villi population, displayed in Figure 6 , have also this periodic 
TABLE 2. Tumour cell population (containment) for a treatment of $2+5$ days.

\begin{tabular}{ccc}
\hline$\tau_{A}$ & $\max _{t} B(t)$ & $\min _{t} B(t)$ \\
\hline 0.4 & 36000 & 21 \\
0.5 & 119000 & 390 \\
0.6 & 352000 & 5900 \\
\hline
\end{tabular}

TABLE 3. Tumour cell population (containment): 2.5 days of treatment +4.5 days of recovery, chonomodulated and equivalent constant treatment.

\begin{tabular}{ccc}
\hline$\tau_{A}$ & $\max _{t} B(t)$ & $\max _{t} B(t)$ \\
infusion & chronomodulated & constant \\
\hline 0.4 & 18400 & 38500 \\
0.5 & 75300 & 129600 \\
0.6 & 247000 & 367000 \\
\hline
\end{tabular}

tendency ensuring that the treatment can be repeated while controlling tumour growth. Table 2 presents the best objective function, $\max _{t} B(t)$ for the best infusion rate $i$, after an initial treatment period of 3 days, for infusion cycle experiments of $2+5$ days and three different values of the minimal admissible fraction of the villi population $\tau_{A}=0.4,0.5$ and 0.6. Table 2 also presents the minimum tumour population reached with this best infusion rate. While the tumour population can be brought to a very low level, due to the exponential growth of the tumour, the maximal number of tumour cells, after a initial period of 3 days, remains quite high, though under control.

Table 3 corresponds to experiments performed in a four weeks course, with an infusion cycle of 2.5 days followed by 4.5 days of recovery. In this particular setting, we also assessed here the gain obtained by the chronomodulated scheme as compared to an equivalent constant infusion scheme: the best objective function, $\max _{t} B(t)$ after a 3 days initial treatment period, is given for the chronomodulated infusion law and for a constant infusion law (applied during the 2.5 days infusion periods) with an infusion rate equal to the mean of the corresponding chronomodulated instantaneous infusion flow.

One should not be surprised that for the same chronomodulated scheme, a weekly containment treatment with an infusion period of 2.5 days leads to a lower level of tumour cells than the same with an infusion period of 2 days: the longer the recovery period, the higher the tumour can grow up. A trade-off has to be found between the length of the necessary recovery period and the admissible maximal level for the tumour. Furthermore, Table 3 also shows the net gain obtained by the chronomodulation strategy: the number of tumour cells is significantly reduced when the treatment takes benefit of the circadian rhythm.

\section{Discussion AND CONCLUSION}

\subsection{Optimisation method}

In this paper, we have detailed an optimisation procedure taking into account objective and constraint functions that are optimised in an $L^{\infty}$ manner, and not according to a quadratic $L^{2}$, or $L^{1}$ criterion, as has already been done before in the literature. To our knowledge, this approach is original, as are the differentiability results stated here. This approach is intended to stick to the actual clinical or experimental problem, and in this respect extreme values are of uppermost importance as they may be fatal for the patient, our $L^{\infty}$ approach controls them whereas $L^{2}$ or $L^{1}$ integrated criteria don't. It is clear however that our mathematical model is still too simple as it should take into account different toxicities (other than the intestinal, such as bone marrow toxicity or sensory neuropathy, as is the case with oxaliplatin in clinical settings). This would lead to more 
equations and more constraints, with possible weighting - left to the clinician - between them, but our approach extends easily to such far more complicated cases.

From the algorithmic point of view, although we cannot assert that our optimisation algorithm reaches a global minimum, the multiplicity of iterations and the storing of the best solution at each iteration, together with the use of some random walk to explore the phase space gives us hints of its actual optimality and robustness.

\subsection{Why a purely deterministic model?}

It is noteworthy that, as stated in the introduction, circadian rhythms in living organisms are synchronised by natural light [and by social rhythms in Humans], which implies - and is observed in experimental and clinical settings - that all subjects show the same periods and time peaks in drug sensitivity. At the cell population level, variabilities in circadian drug sensitivity phases may occur in each compartment, healthy or tumoral, but both phases are synchronised by the central pacemaker located in the hypothalamus (suprachiasmatic nuclei). These phases and their variabilities may be assessed at the peripheral tissue level by measuring clock gene (Per2, Clock, Bmal1) expression or clock-controlled gene expression of apoptosis proteins such as BAX or BCL2 as a function of circadian time [19], and at the central level by body rhythm recordings, such as temperature. Taking them into account would only add a new parameter to the model: the robustness of circadian control. This will surely be of interest in a future model, for describing differences in the quality of individual circadian synchronisation within a population of individuals (laboratory animals or patients). But this was not our primary concern in setting this frame for presenting the optimisation procedure. In the same way, artificially adding stochastic components to the model would hardly be of any interest for this presentation.

\subsection{Limits of this model}

The model we used addresses the optimisation of cancer chemotherapy using pharmacokineticpharmacodynamic and circadian modelling with a toxicity constraint. It does not address the problem of drug resistance and its evolution in tumour cell populations, which is also an important limitation in cancer chemotherapy, and has been studied by various authors already $[15,25,33]$. Nor does it address the fact that most anti-cancer drugs (but not oxaliplatin, to our knowledge) are known to show cell cycle phase specificities. The former should be taken into account by an extension of our simple model to subpopulations for tumour growth, quiescent, proliferative drug-sensitive and proliferative drug-resistant. The latter implies future modelling of cell cycle progression and apoptosis at the tissue level for tumour cell populations, with coupling to local circadian clocks - a coupling which has been shown experimentally to be unidirectional, i.e. control of cell proliferation by the clock, at least for the regenerating liver in mice $[28,32]$. These approaches are complementary, and according to the particular tumour and drug involved, partial or extended models should be used for chemotherapy optimisation.

\subsection{Model identification and future clinical applications}

We must stress that the numerical results presented are highly dependent on the values of parameters used, some of which had to be grossly estimated in our dataset - besides, the dataset should be completely different for other tumours and other cytotoxic drugs -, so that any generalisation of these results to clinical conclusions would at this stage be completely hazardous. Yet, as far as equivalent modelling equations and their parameters may be identified in other settings, the same optimisation procedure could be successfully applied.

We will not hide that identifying cell and tissue pharmacokinetics and pharmacodynamic dose-effects (even if these two aspects are merged together) means acquiring knowledge of hidden mechanisms which are not easily accessible in everyday clinical routine, and this remains today a shortcoming of the method proposed here. Nevertheless, we have reasonable hopes that such data, provided by the development of modern pharmacokineticspharmacodynamics (PK-PD) and molecular biology techniques, will become more and more available in the next future.

This in our meaning justifies developing such optimisation methods which make it necessary to model intimate tissue mechanisms for drug efficacy and toxicity effects. Whole organism modelling and PK-PD development 
could lead us to use other observable than cell population numbers - for instance, as far as oxaliplatin is concerned, peripheral sensory neuropathy has recently been proposed to be linked to insults to nucleoli in nerve ganglia [29], not measurable by cell kill evaluations-, but such future, more detailed, models will still be liable for the optimisation method we presented here.

Finally, one may notice that it gives a rationale, not only for circadian chronotherapy theoretic studies which first motivated it, but also, using optimisation in the product space $L^{2}\left(\left[t_{0}, t_{f}\right]\right) \times\left[t_{0}, t_{f}\right]$, for so-called intensive therapies, in which the stress is put on the best treatment course and between courses durations making possible a necessary increase in the delivered dose when classical therapeutic schemes have failed.

Acknowledgements. This work was supported by the EU Network of Excellence BIOSIM, contract 005137.

\section{REFERENCES}

[1] Z. Agur, R. Arnon and B. Schechter, Effect of the dosing interval on myelotoxicity and survival in mice treated by cytarabine. Eur. J. Cancer 28A (1992) 1085-1090.

[2] L.K. Andersen and M.C. Mackey, Resonance in periodic chemotherapy: a case study of acute myelogenous leukemia. J. Theor. Biol. 209 (2001) 113-130.

[3] J.F. Bonnans, J.C. Gilbert, C. Lemarechal and C.A. Sagastizabal, Numerical Optimization: Theoretical and Practical Aspects. Springer Universitext (2003).

[4] N.A. Boughattas, F. Lévi, et al., Circadian Rhythm in Toxicities and Tissue Uptake of 1,2-diamminocyclohexane(trans1)oxaloplatinum(II) in Mice. Cancer Research 49 (1989) 3362-3368.

[5] N.A. Boughattas, B. Hecquet, C. Fournier, B. Bruguerolle, A. Trabelsi, K. Bouzouita, B. Omrane and F. Lévi, Comparative pharmacokinetics of oxaliplatin (L-OHP) and carboplatin (CBDCA) in mice with reference to circadian dosing time. Biopharmaceutics and drug disposition 15 (1994) 761-773.

[6] N.F. Britton, N.A. Wright and J.D. Murray, A mathematical model for cell population kinetics in the intestine. J. Theor. Biol. 98 (1982) 531-541.

[7] L. Canaple, T. Kazikawa and V. Laudet, The days and nights of cancer cells. Cancer Research 63 (2003) 7545-7552.

[8] J. Clairambault, D. Claude, E. Filipski, T. Granda and F. Lévi, Toxicité et efficacité antitumorale de l'oxaliplatine sur l'ostéosarcome de Glasgow induit chez la souris : un modèle mathématique. Pathologie-Biologie 51 (2003) 212-215.

[9] L. Cojocaru and Z.A. Agur, Theoretical analysis of interval drug dosing for cell-cycle-phase-specific drugs. Math. Biosci. 109 (1992) 85-97.

[10] B.F. Dibrov, M.A. Zhabotinski, Yu.A. Neyfakh, M.P. Orlova and L.I. Churikova, Mathematical model of cancer chemotherapy. Periodic schedules of of phase-specific cytotoxic agent administration increasing the selectivity of therapy. Math. Biosci. 73 (1985) 1-34.

[11] B.F. Dibrov, Resonance effect in self-renewing tissues. J. Theor. Biol. 192 (1998) 15-33.

[12] L. Edelstein-Keshet, Mathematical Models in Biology. NY: McGraw-Hill (1988) 210-270.

[13] A.W. El-Kareh and T.W. Secomb, A mathematical model for cisplatin cellular pharmacodynamics. Neoplasia 5 (2004) 161-169.

[14] S. Faivre, D. Chan, R. Salinas, B. Woynarowska and J.M. Woynarowski, DNA Single Strand Breaks and apoptosis induced by oxaliplatin in cancer cells. Biochemical Pharmacology 66 (2003) 225-237.

[15] K.R. Fister and J.C. Panetta, Optimal control applied to cell-cycle-specific cancer chemotherapy. SIAM J. Appl. Math. 60 (2000) 1059-1072.

[16] L. Fu, H. Pellicano, J. Liu, P. Huang and C.C. Lee, The Circadian Gene Period2 Plays an Important Role in Tumor Suppression and DNA Damage Response In Vivo. Cell 111 (2002) 41-50.

[17] L. Fu and C.C. Lee, The circadian clock: pacemaker and tumour suppressor. Nature Reviews 3 (2003) 351-361.

[18] T.G. Granda, R.-M. D'Attino, E. Filipski, et al., Circadian optimisation of irinotecan and oxaliplatin efficacy in mice with Glasgow osteosarcoma. Brit. J. Cancer 86 (2002) 999-1005.

[19] T.G. Granda, X.H. Liu, R. Smaaland, N. Cermakian, E. Filipski, P. Sassone-Corsi and F. Levi, Circadian regulation of cell cycle and apoptosis proteins in mouse bone marrow and tumor. FASEB J. 19 (2005) 304.

[20] M. Gyllenberg and G.F. Webb, Quiescence as an explanation of gompertzian tumor growth. Growth, Development and Aging 53 (1989) 25-33.

[21] M. Gyllenberg and G.F. Webb, A nonlinear structured population model of tumor growth with quiescence. J. Math. Biol. 28 (1990) 671-694.

[22] M.H. Hastings, A.B. Reddy and E.S. Maywood, A clockwork web: circadian timing in brain and periphery, in health and disease. Nat. Rev. Neurosci. 4 (2003) 649-661.

[23] A. Iliadis and D. Barbolosi, Optimising drug regimens in cancer chemotherapy by an efficacy-toxicity mathematical model. Computers Biomed. Res. 33 (2000) 211-226. 
[24] A. Iliadis and D. Barbolosi, Optimising drug regimens in cancer chemotherapy: a simulation study using a PK-PD model. Computers Biol. Med. 31 (2001) 157-172.

[25] M. Kimmel and A. Swierniak, Using control theory to make cancer chemotherapy beneficial from phase dependence and resistant to drug resistance. Technical report \#7, Ohio State University, Nov. 2003, available on line at http://mbi.osu.edu/publications/techreport7.pdf (2003).

[26] F. Lévi, G. Metzger, C. Massari and G. Milano, Oxaliplatin: Pharmacokinetics and Chronopharmacological Aspects. Clin. Pharmacokinet. 38 (2000) 1-21.

[27] F. Lévi (Ed.), Cancer Chronotherapeutics. Special issue of Chronobiology International 19 \#1 (2002).

[28] T. Matsuo, S. Yamaguchi, S. Mitsui, A. Emi, F. Shimoda and H. Okamura, Control mechanism of the circadian clock for timing of cell division in vivo. Science 302 (5643) (2003) 255-259.

[29] M.C. McKeage, T. Hsu, G. Haddad and B.C. Baguley, Nucleolar damage correlates with neurotoxicity induced by different platinum drugs. Br. J. Cancer 85 (2001) 1219-1225.

[30] M. Mishima, G. Samimi, A. Kondo, X. Lin and S.B. Howell, The cellular pharmacology of oxaliplatin resistance. Eur. J. Cancer 38 (2002) 1405-1412.

[31] C.S. Potten and M. Loeffler, Stem cells: attributes, cycles, spirals, pitfalls and uncertainties. Lessons for and from the crypt. Development 110 (1990) 1001-1020.

[32] U. Schibler, Circadian rhythms. Liver regeneration clocks on. Science 302 (5643) (2003) 234-235.

[33] G. Swan, Role of optimal control theory in cancer chemotherapy. Math. Biosci. 101 (1990) 237-284.

[34] G.F. Webb, Resonance phenomena in cell population chemotherapy models. Rocky Mountain J. Math. 20 (1990) $1195-1216$.

To access this journal online: www.edpsciences.org 